\title{
Estimating the Low Wind Effect on SPHERE with experimental and on-sky data
}

\author{
Masen Lamb ${ }^{a, b}$, Carlos Correia ${ }^{c}$, Jean-François Sauvage ${ }^{c, d}$, Jean-Pierre Véran $^{b}$, David \\ Andersen $^{b}$, \\ ${ }^{a}$ University of Victoria, 3800 Finnerty Rd, Victoria, Canada; \\ ${ }^{b}$ NRC Herzberg Astronomy, 5071 W. Saanich Rd, Victoria, Canada; \\ ${ }^{c}$ Aix Marseille Université, CNRS, LAM (Laboratoire d'Astrophysique de Marseille) UMR \\ 7326, 13388, Marseille, France; \\ ${ }^{d}$ ONERA, 29 Avenue de la Division Leclerc, 92320 Châtillon, France;
}

\begin{abstract}
In nights at Cerro Paranal where good seeing and low wind conditions are present, the PSF delivered to the focal plane of the SPHERE instrument has been shown to have significant errors, and have been aptly described as the 'Low Wind Effect' (LWE). We demonstrate here a method to quantify the LWE using experimental and on-sky data. We find single image phase diversity is a useful tool in quantifying the LWE and can be used to monitor this effect over the course of the night.
\end{abstract}

Keywords: Phase Diversity, Wavefront Sensing, Adaptive Optics

\section{INTRODUCTION}

The 'low wind effect' on the VLT/SPHERE system describes an effect that typically occurs on a night with good seeing and low wind at Cerro Paranal whereby the PSF is significantly degraded. The effect occurs roughly one in five nights and can drive the SPHERE system to complete closure for the night. The effect has been explored in an attempt to establish both its nature and possible forms of correction (i.e. [1]). One approach to correcting the effect is through an accurate quantification and subsequent offset on the deformable mirror (DM). If such an approach is to be entertained, then the quantification method must be relatively quick and accurate. It is thought that the wavefront sensor (WFS) used by SPHERE is potentially incapable of quantifying this effect, which would explain why the effect is not corrected for by the adaptive optics (AO) system in the first place. This may be due to the inabilities of the Shack-Hartmann WFS to detect differential piston ([1]); therefore quantifying the wavefront from the focal plane is desirable.

Lamb et al. 2017 [2] showed that phase diversity is a suitable choice to estimate the LWE and furthermore, that a single DTTS image is enough to quantify the LWE. We now wish to validate these techniques outside the scope of simulation, therefore we turn our investigation to both an experimental bench and on-sky data. The on-sky data were acquired over the course of a night subject to a strong LWE. Showing this validation is essential prior to attempting any sort of real time estimation or correction at the telescope. In addition, exploring the evolutionary effects over the course of a night subject to the LWE may lead to valuable insight in the approach to be taken to estimate this effect in real time. For example, if the effect is shown to have features which are relatively constant (i.e. on the order of minutes to hours) then developing an algorithm that needs to operate extremely quickly may not be necessary. Therefore the scope of this paper is to: (i) first demonstrate the Phase Diversity techniques explored in [2] can effectively estimate the LWE on an experimental bench; (ii) apply these techniques on a set of data acquired on SPHERE during a night subject to the LWE to try and quantify this effect; and (iii) explore the evolution of the effect over the course of the night. These three exercises will allow us to consider quantification at the telescope itself during a night with such an effect, and additionally provide insight as to how often one would need to measure the effect if they were to consider applying some sort of real-time correction (i.e. by providing offset slopes to the $\mathrm{AO}$ system).

Further author information: M.L.: E-mail: masen@uvic.ca 


\section{METHODS AND OBSERVATIONS}

\subsection{Mithic Experimental Bench Observations}

To validate our Phase Diversity technique we estimate the phase on an experimental bench with a known phase error. In particular, we consider two different scenarios on a high-contrast imaging experimental bench called MITHIC, located at Laboratoire d'Astrophysique de Marseille. On the bench we consider a phase map pertaining to $44 \mathrm{~nm}$ RMS WFE of 20 randomly oriented Zernike Polynomials injected into the system via a spatial light modulator (SLM), and a phase screen with $20 \mathrm{~nm}$ RMS WFE representative of the LWE phase. The phase screen in the latter case is described in detail in [3]. Figure 1 displays the phase in each scenario: the left panel is the phase map introduced by the SLM while the right panel is a measurement of the phase screen taken with a ZELDA WFS [4].
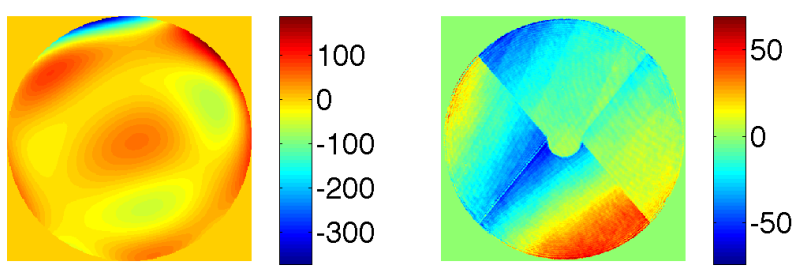

Figure 1. Phase maps representing each scenario considered on our experimental bench. Left: $44 \mathrm{~nm} \mathrm{rms}$ WFE phase map resulting from random coefficients applied to the first 20 Zernike Polynomials; this phase map is projected into our system via a SLM. Right: phase screen with an imprint of a representation of the LWE ( $20 \mathrm{~nm} \mathrm{rms}$ WFE), inserted in the pupil plane of the system.

For each phase scenario five images were acquired with amplitudes of focus, corresponding to $0,50,75,100$, and $150 \mathrm{~nm}$ RMS. Figure 2 shows these images on a log-scale. This data will be utilized with Phase Diversity in Section 2.3 in an attempt to estimate the phase of each scenario.
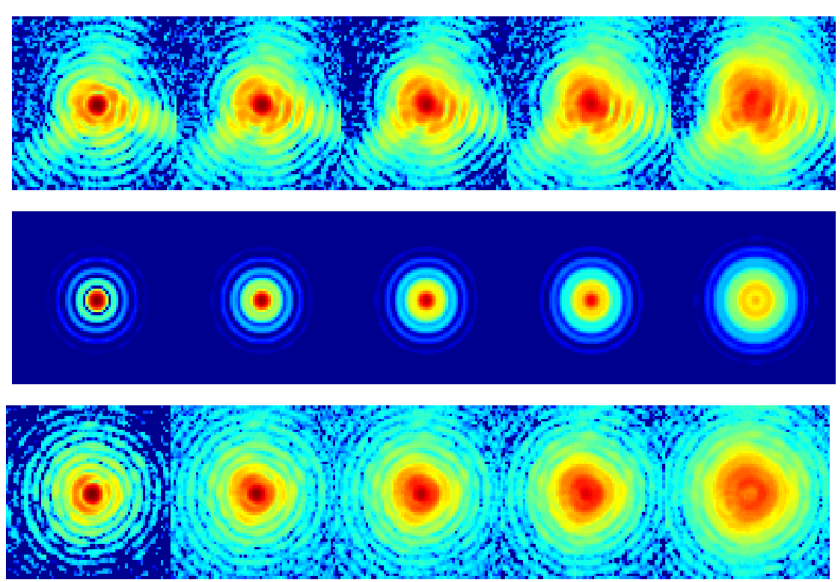

Figure 2. Top: images obtained at 5 different focal plane positions in the scenario where $44 \mathrm{~nm}$ rms WFE is injected from 20 random Zernike Polynomials; the focal positions are 0, 50, 75, 100 and $150 \mathrm{~nm}$ rms (from left to right). Middle: synthetic images created using the known bench parameters at the same focal positions. The real and synthetic images are used in the Phase Diversity algorithm to estimate the phase of the system. Bottom: images obtained in the same manner using the LWE phase screen. All images are shown here in log-scale. The images were created with a $677 \mathrm{~nm}$ fiber source.

\subsection{On-Sky Data}

On-sky data was acquired on the Differential Tip Tilt Sensor (DTTS) on the VLT SPHERE adaptive optics system during a night largely affected by the LWE in October 2016. A total of 13577 images were recorded every 
two seconds, spanning $\sim 4$ hours of a night. Figure 3 displays DTTS images affected by the LWE at three different times throughout the course of this sequence. The images were acquired in H-band with a sampling of 3.46 pixels across the full width at half maximum. The size of the images are $32 \times 32$ pixels, resulting in PSF information-loss at the outer regions of the images; this lost information is less impactful on any focal-plane wavefront sensing due to the effect of pupil apodization, which already suppresses high spatial frequencies. Therefore if the PSF is properly modelled (i.e. correct pupil apodization, spider location, pixel scale, etc.), then Phase Diversity should have the capability to sense wavefront aberrations from DTTS images. In Section 3, we apply the techniques developed on the MITHIC bench in an attempt to estimate the LWE from the DTTS images and discuss our results.
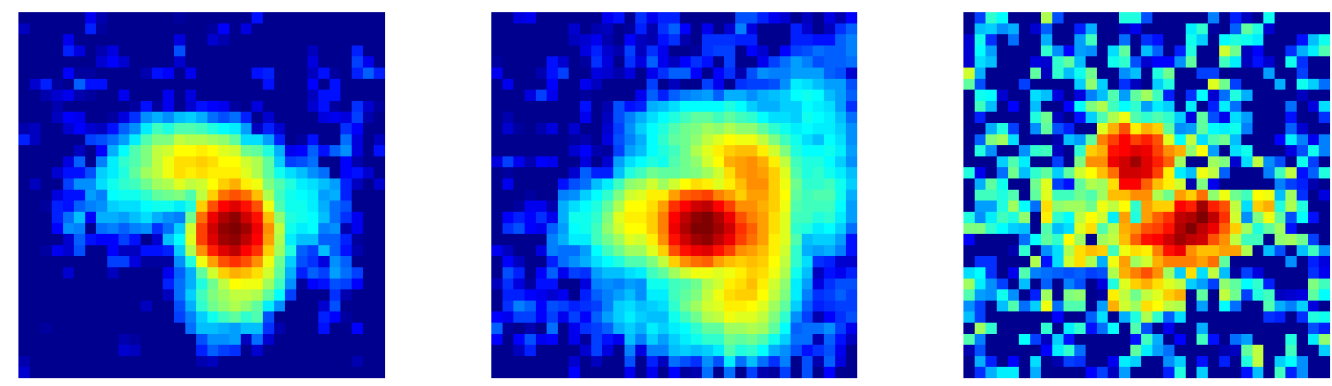

Figure 3. Images obtained by the DTTS on SPHERE at three different times affected by the LWE on this particular night.

\subsection{Evaluating MITHIC Data}

\subsection{Classic Phase Diversity}

We first employ 'Classic Phase Diversity' using data taken from the MITHIC experimental bench. We consider Classic Phase Diversity as the scenario similar to that originally put forth by [5] and [6], specifically where an in-focus image and an out-of-focus image are used to estimate the coefficients of a modal basis representative of the phase error in question; the details of our approach are summarized in [7]. This estimation is performed in two scenarios: with and without object estimation. As previously shown in Chapter 4, Phase Diversity with object estimation is best achieved when one of the images is in-focus; conversely, we showed that Phase Diversity with no-object estimation (the object is assumed a point source) ideally uses diverse images away from the focal plane, because including an in-focus image degrades the phase estimation. In the following sections we employ Classic Phase Diversity with and without object estimation to quantify known phase errors injected into our system. We attempt to recover these known errors in order to validate our techniques, which will then be used to consider more complicated examples.

\subsubsection{Intrinsic NCPA}

The intrinsic NCPA of the MITHIC bench must first be quantified so that its signature can be removed from future estimations where we estimate induced phase injections. The intrinsic NCPA are estimated with Phase Diversity in two scenarios: object estimation using an in-focus image and $150 \mathrm{~nm}$ rms focus image, and no object estimation (assuming a point-source) using the 100 and $150 \mathrm{~nm}$ rms focus images. The synthetic images created in the Phase Diversity algorithm here assume a uniform, circular pupil; in all future phase estimates using MITHIC data we continue to make this assumption. Figure 4 displays the phase estimated from the object-estimation scenario, along with a reconstructed PSF from this phase. Also shown is the in-focus PSF for comparison; visually the PSFs agree. The rms of the estimated intrinsic NCPA is 16 and $17 \mathrm{~nm} \mathrm{rms}$ for the object and no-object estimation cases, respectively.

\subsection{Estimating a Known Phase Injection}

As previously discussed, the known phase injected (see Figure 1, left panel) produced the images shown in Figure 2 (top). We now use these images with the same Phase Diversity approach described in 2.4 to estimate this phase injection. We estimate coefficients for seven radial orders of Zernike Modes using both Phase Diversity 

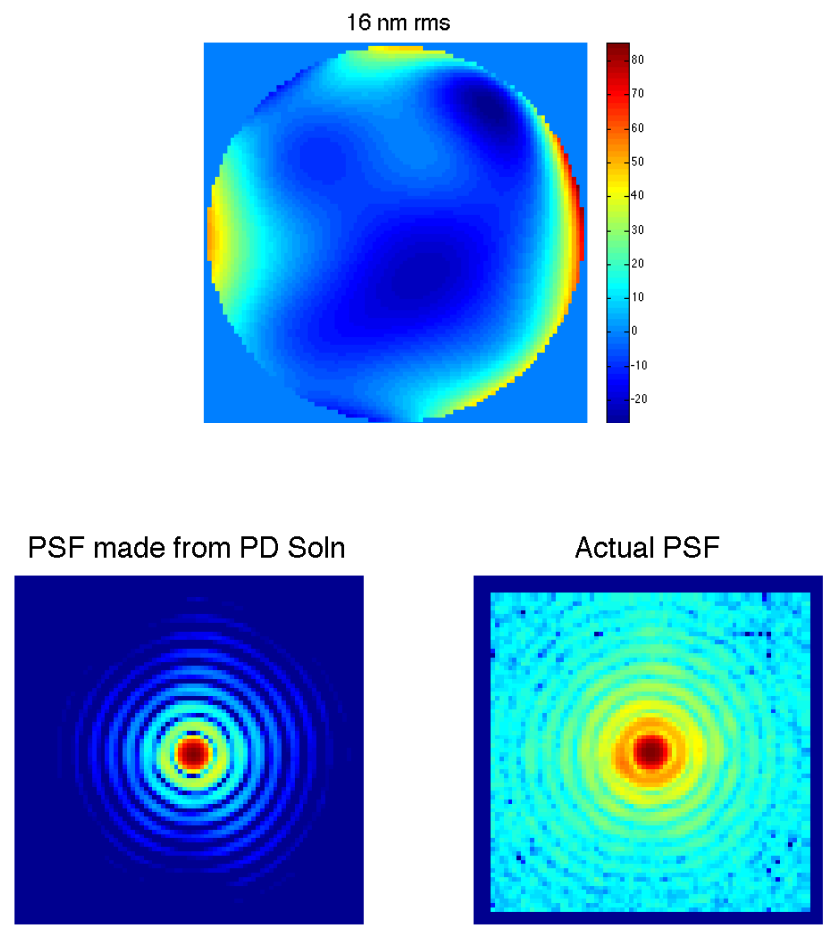

Figure 4. Top: Estimated intrinsic NCPA phase from the reconstruction of 7 radial orders of Zernike Polynomials; the Zerniikes were estimated employing classic Phase Diversity with two images: the in-focus and largest defocussed image. In this case the object was also jointly estimated. Bottom: reconstructed PSF using the estimated phase (left) and the actual in-focus PSF (right); visually the reconstructed PSF is nearly identical to the actual PSF. This estimate of the phase represents the intrinsic NCPA of the system and must be subtracted from any future scenario when trying to estimate a known phase injection.

scenarios (object/no-object estimation). The phase maps are reconstructed from the Zernike coefficients and the signature of the intrinsic NCPA is removed. Figure 5 shows our estimated phase for both scenarios, along with: the actual phase WFE, the SH-WFS phase WFE and the residual between our estimate and the actual. The residual phase maps are $10 \mathrm{~nm}$ RMS under both Phase Diversity scenarios. The reconstructed PSF from the object-estimation scenario is shown in Figure 6, which from visual inspection agrees with the actual in-focus PSF.

\subsection{LWE Phase Screen}

We now utilize the same approach described in the previous Section to estimate the LWE phase, provided by a phase screen in the pupil plane. In this case we employ the basis described in [2] in lieu of Zernike Polynomials. The intrinsic NCPA are now estimated using this basis (following the same manner in Section 2.4.1) so that they can be removed from future LWE phase screen estimations using this basis. This ensures any contribution of the intrinsic NCPA to the estimated basis in the presence of the LWE phase screen will not be contaminated. The resulting basis coefficient estimates from both Phase Diversity scenarios are reconstructed into phase maps (and removed for intrinsic NCPA) and shown in Figure 7. From inspection it can be seen the general trend of the actual phase (right) is reproduced in both of the estimates (left, middle), and the rms WFE is nearly identical. The residual between the estimated and actual phase of the top quadrant is on the order of a few $\mathrm{nm}$ rms, and a slight variation in tip/tilt can cause the visual discrepancy shown here. Figure 8 displays a reconstruction of the PSF from the phase estimated in one of the scenarios (left); it is nearly identical to the actual in-focus PSF (right). We conclude that we can make an accurate phase estimation of the LWE phase screen. 

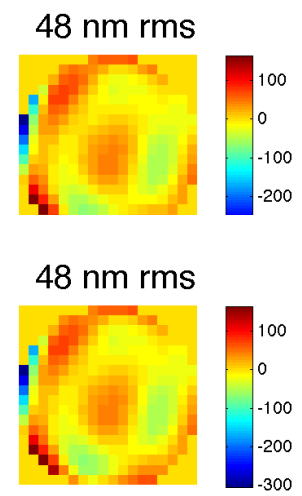

$33 \mathrm{~nm}$ rms

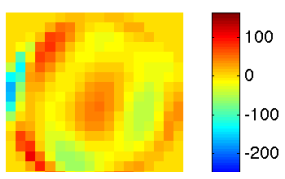

$33 \mathrm{~nm}$ rms

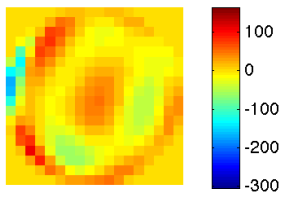

$33 \mathrm{~nm} \mathrm{rms}$

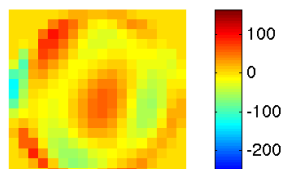

$29 \mathrm{~nm}$ rms

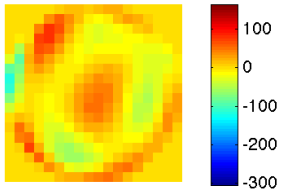

$10 \mathrm{~nm} \mathrm{rms}$

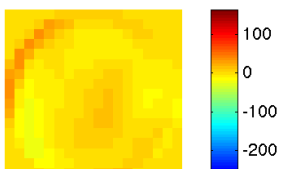

$10 \mathrm{~nm} \mathrm{rms}$

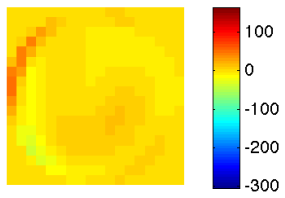

Figure 5. Top: estimation of the known phase-injection using Classic Phase Diversity with object estimation. Bottom: estimation of the same phase with no-object estimation (assuming a point source). From left to right: SH-WFS phase measurement, phase injected into system, estimated phase (via 7 radial orders of Zernikes), residual between the actual and estimate. The Phase Diversity described here used the 4 defocussed images from the sample, assuming a point source. For both cases the intrinsic NCPA were removed from the estimated phase. Note: the resolution of the phasemaps are restricted by that of the SH-WFS (18x18).
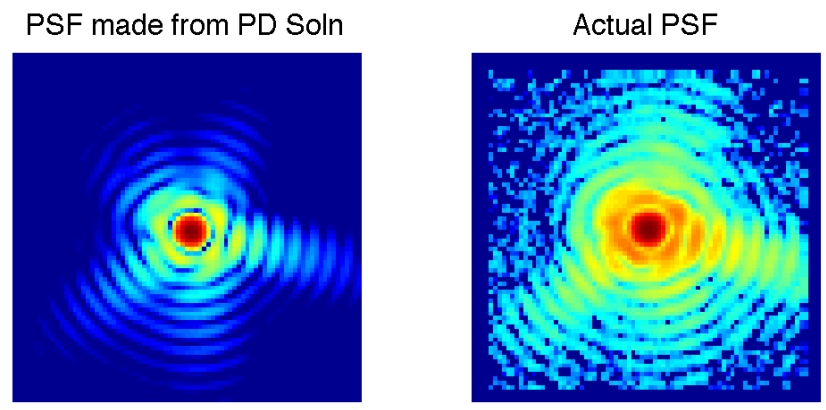

Figure 6. PSF comparison showing the reconstructed PSF from the estimated phase (left) with the actual in-focus PSF (right) for the images created from the $44 \mathrm{~nm}$ RMS phase map (injected by the SLM). The phase was estimated here using Classic Phase Diversity with object estimation. Visually the estimated PSF is very similar to the actual PSF.

\subsubsection{Single Image Phase Diversity}

Employing Classic Phase Diversity on-sky has proved to be difficult in the past (i.e. [8]). Quantifying the LWE from on-sky data using this approach will undoubtedly present equivalent difficulties, one of these difficulties being the fact that the evolution between two images on-sky (such as in and out of focus images used with Phase Diversity) can yield errors contaminating the true signal of the LWE. It is thus desirable to consider single image Phase Diversity, which can be achieved if the object is assumed a point-source and enough focus is used as the diversity. We follow the same procedure as the simulation shown in [2], where the SPHERE/DTTS imager small focus error ( $\sim 20 \mathrm{~nm} \mathrm{rms})$ was used as diversity. We explore here the possibility of estimating the wavefront from single images acquired by the DTTS during a night that exhibited the LWE. Prior to this, however, we aim to validate the technique of single image Phase Diversity on the experimental setup described here with the MITHIC bench.

A single image on the bench is considered using the largest focus diversity (150 nm rms) in both phase scenarios (known phase injection and LWE phase screen). We assume the object is a point source and estimate the phase in the exact same manner as Sections 2.5 and 2.6. Figure 9 displays the estimated phases for each scenario: the known phase injection and LWE phase screen is estimated to almost the exact same degree as 

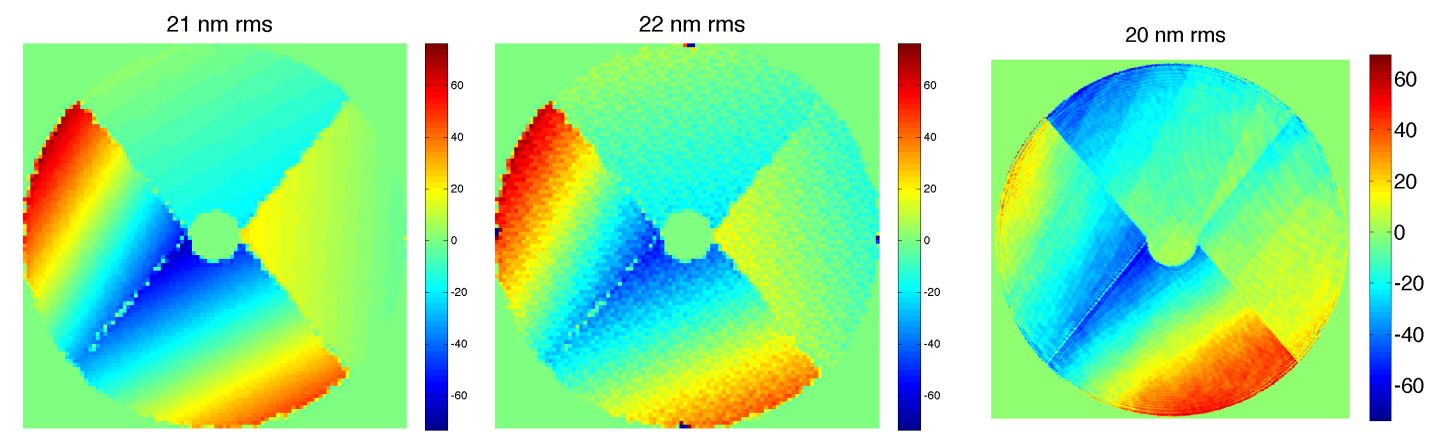

Figure 7. Phase estimates (no object estimation - left, object estimation - middle) compared with the actual phase (right). The general features in the estimation reproduce the known phase for three of the four segments. The rms WFE of the estimation is nearly identical to that of the phase screen.
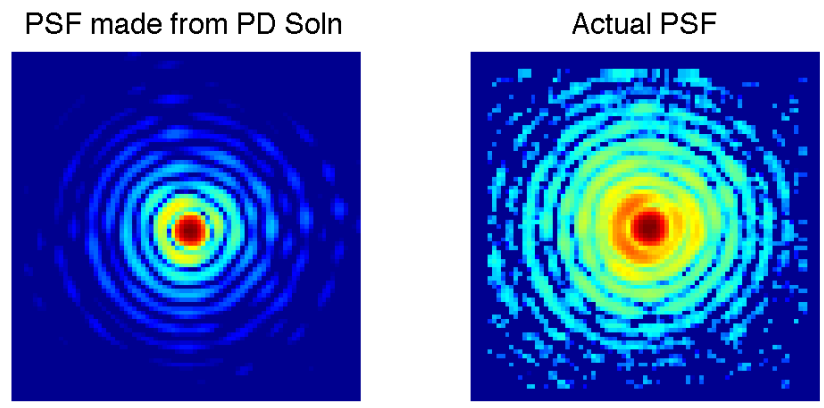

Figure 8. PSF comparison showing the reconstructed PSF from the estimated phase (left) with the actual in-focus PSF (right) using the LWE phase screen on the MITHIC bench. The phase was estimated here using Classic Phase Diversity with object estimation. Visually the estimated PSF is very similar to the actual PSF.

Classic Phase Diversity (shown in Figures 5 and 7), and the residual between the estimate of the phase screen and that measured by Zelda is $\sim 12 \mathrm{~nm}$ RMS (see Figure 10). From these results we are confident in the technique of single image Phase Diversity for other applications. In the following Sections we use this technique to estimate the LWE with single DTTS images on the SPHERE system during an active LWE night.
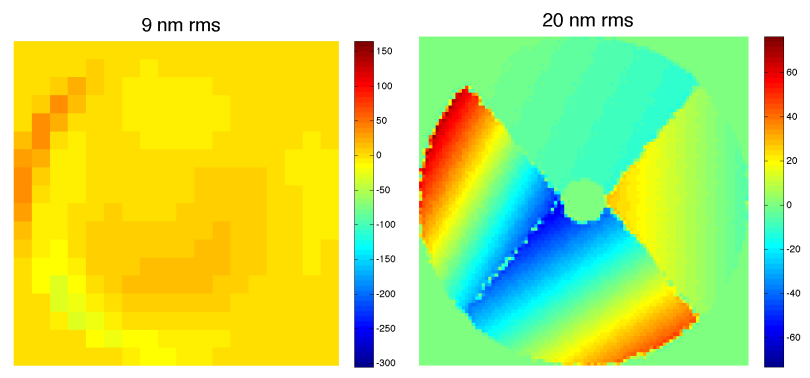

Figure 9. Single image Phase Diversity estimates of both the known phase injection (left, residual difference between estimate and actual shown) and the LWE phase screen (right). The residual phase in the known phase injection scenario is nearly identical to that estimated with Classic Phase Diversity (see Figure 5). Similarly, the LWE phase is nearly identical to its Classic Phase Diversity esimation counterpart (see Figure 7). 


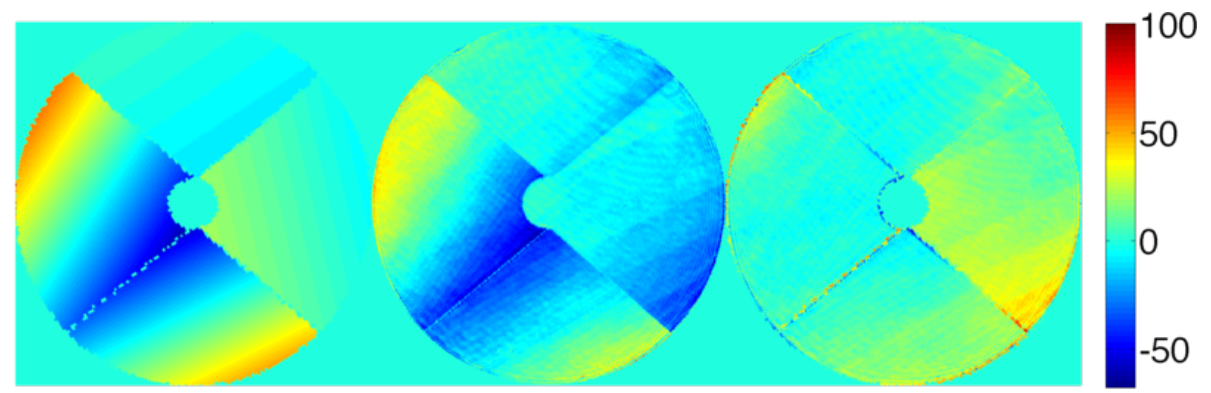

Figure 10. Single image Phase Diversity estimates of the LWE phase screen (left) and the actual phase screen (middle) as measured by Zelda. The residual between the two is shown on the right. The rms WFE of each phase map is 18,20 and $12 \mathrm{~nm}$, respectively.

\section{EVALUATING ON-SKY DATA}

Using the DTTS images discussed in Section 2.3 we employ single image Phase Diversity. The synthetic PSFs used in the algorithm are created from the known SPHERE apodization and VLT pupil, shown here in Figure 11. The DTTS imager is inherently subject to $20 \mathrm{~nm}$ RMS defocus inherent in the design of the DTTS, and we take advantage of this designed focus as diversity for our algorithm.
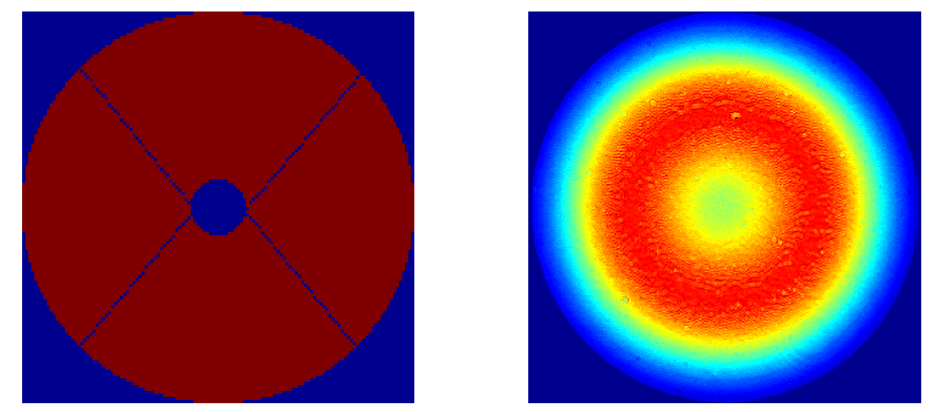

Figure 11. Pupil model (left) and apodization (right) used to model the synthetic PSFs used in the Phase Diversity determination of the LWE.

\subsection{Basis selection}

As previously discussed and shown in [2], the choice of basis for our estimation should reflect the nature in which the phase on the pupil is being manipulated during a LWE sequence. To ensure our custom piston/tip/tilt basis (described in [2]) is a reasonable choice, we estimate the LWE on a strongly affected image using both a Zernike and piston/tip/tilt basis and compare. Figure 12 shows the estimation from Phase Diversity using both bases; the Zernike coefficients (estimated from 12 radial orders) are clearly attempting to replicate a phase discontinuity defined by the pupil spiders as shown in the right panel of the Figure. This immediately suggests that part of the LWE is indeed due to the phase discontinuities originating from the secondary spiders. The left panel shows the piston/tip/tilt basis is a simplification of the effect and can naturally accommodate the pupil discontinuities. Furthermore, this twelve mode basis requires significantly less computation than when the large number of Zernike polynomials required to approximate these discontinuities are used. These results validate our choice to estimate the phase with a piston/tip/tilt basis, and we do so for the rest of this Section.

\subsection{Estimation of a strong LWE sequence}

We now turn to a one-minute sequence of images subject to a strong LWE to try and understand the evolution of this effect on short time scales. A strong motivation for this analysis is to assess the capability of this technique 

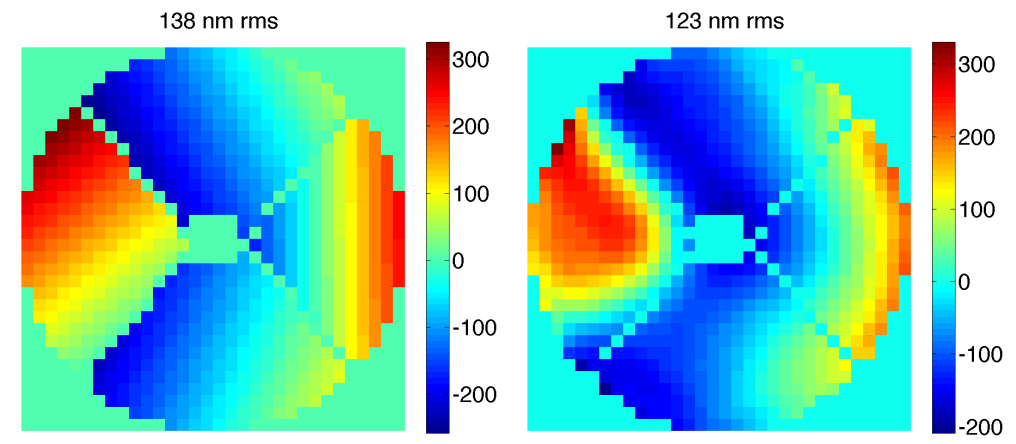

Figure 12. Phase estimated with Phase Diversity on the image shown in Figure 3 (middle) using the piston/tip/tilt basis (left) and 12 radial orders of Zernike polynomials (right). The phase appears to be well approximated with the piston/tip/tilt basis when compared with the Zernike estimate. Furthermore, the Zernike estimate reveals the phase is inherently split into the quadrants defined by the spiders in the pupil. This lends further support for using the piston/tip/tilt basis, which can facilitate the pupil discontinuities between two quadrants.
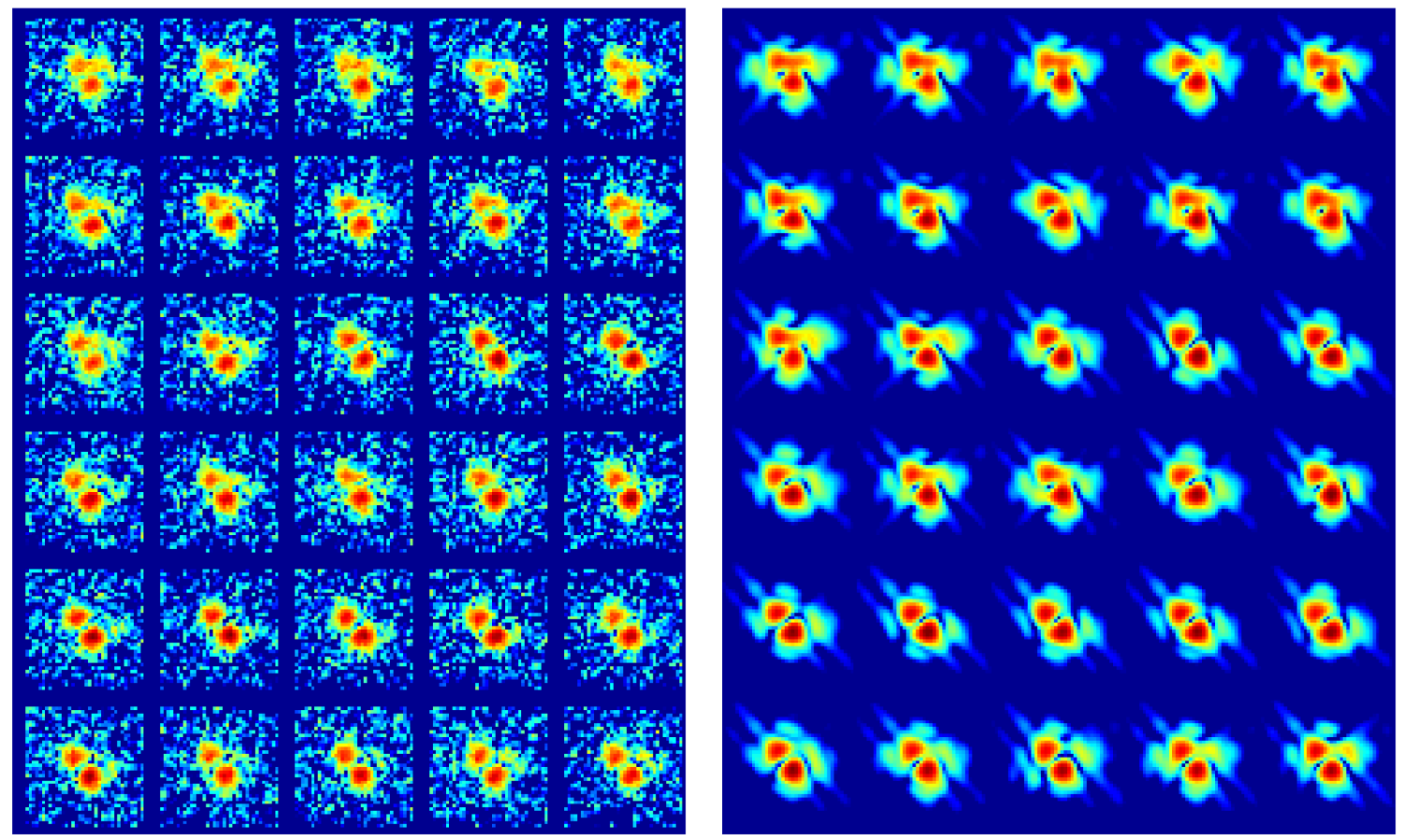

Figure 13. Left: a sequence of DTTS images taken during a particular sequence of the LWE during the night; the images are taken 2 seconds apart and span a total length of approximately one minute. Bottom: reconstructed images from the phase of the LWE estimated by Phase Diversity.

to quickly identify the error associated with the LWE when it happens, and understand how long of a 'window' exists for applying some sort of correction.

The LWE is estimated with Phase Diversity for thirty consecutive DTTS images, using the piston/tip/tilt basis. Figure 13 displays the observed DTTS images, along with PSFs reconstructed from the Phase Diversity estimation; this estimation is shown in Figure 14. The general PSF features over the course of one minute show some minor evolution, which can also be seen in the estimated phase maps. The average P-V WFE over the sequence is $474 \mathrm{~nm}$ while the standard deviation is $62 \mathrm{~nm}$; the P-V phase estimates of the first and last DTTS image are 390 and $508 \mathrm{~nm}$, respectively. 


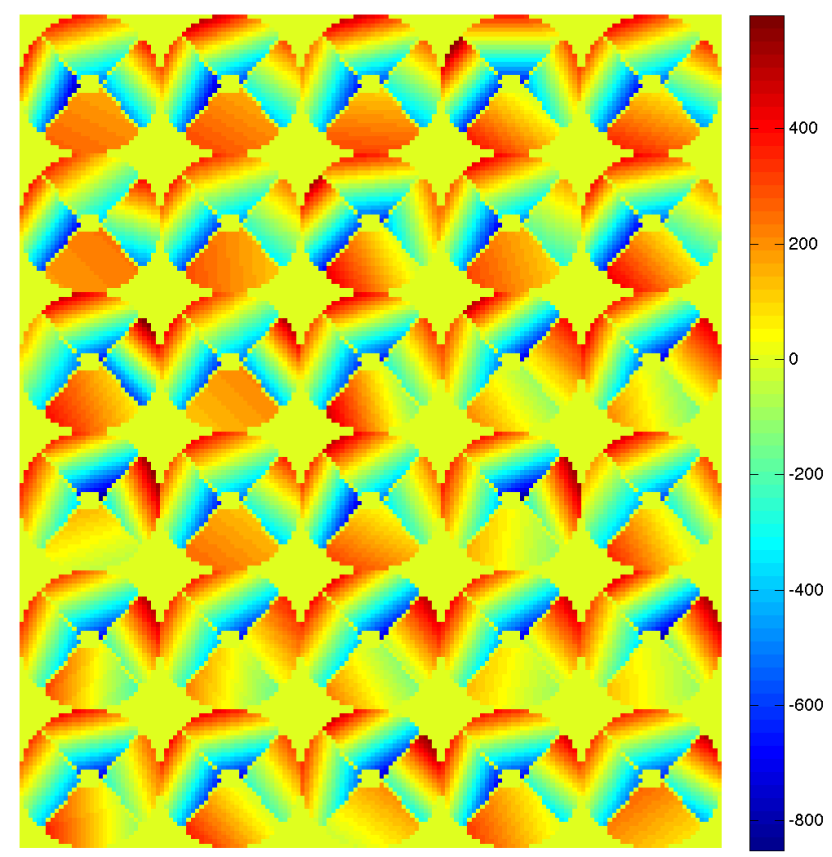

Figure 14. Phase estimates of the LWE during the sequence shown in Figure 13. The mean and standard deviation of the PV phase during this sequence is 474 and $62 \mathrm{~nm}$, respectively. The total evolution of this sequence is about 60 seconds; the first and last PV phase measurements are 390 and $508 \mathrm{~nm}$, respectively.

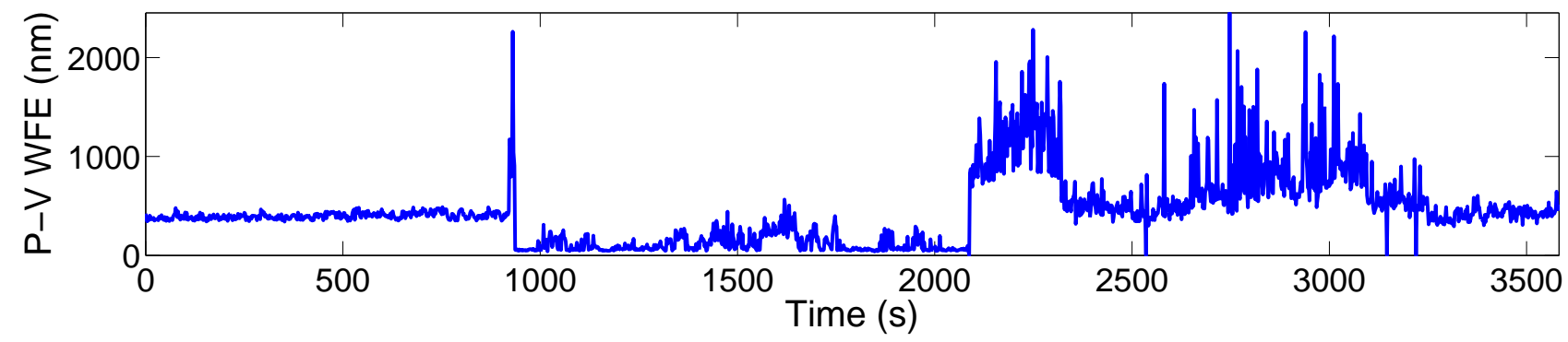

Figure 15. Peak-to-valley phase estimates plotted as function of time for a strong LWE sequence, taken over the course of approximately one hour. There appears to be three distinct episodes of the LWE, each with different strengths.

\subsubsection{Evolution of the LWE over one hour}

It is now worth considering how the LWE behaves on a time-scale much longer than the course of one minute to grasp its evolutionary behaviour. We consider a sequence of images obtained over a period of approximately one hour. The phase is estimated for each image in the sequence and its P-V WFE is plotted as a function of time (Figure 15). Upon inspection of this Figure, several interesting features become immediately apparent. First, there seems to be three distinct 'episodes' of the LWE, suggesting a particular event of some sort taking place at two different times over this sequence. Second, during each 'episode' (or event), the variation of the LWE is different. The first episode shows a very steady WFE, the second episode shows varying LWE strength between 0 and $400 \mathrm{~nm}(\mathrm{P}-\mathrm{V})$, while finally the third episode contains the largest LWE variations with peak amplitudes of $2000 \mathrm{~nm}$. Finally, throughout the latter two episodes, the LWE strength shows that there is an evolving trend (i.e. any measurement will be in relatively close proximity to an adjacent measurement, except in the case of an 'event'). The nature of these events are unclear and are discussed in Section 4. 


\section{DISCUSSION AND CONCLUSIONS}

In the context of SPHERE, the LWE was estimated using single images on the DTTS imager on a night subject to a strong LWE. The techniques developed to do this were first validated on an experimental bench, and then subsequently applied to the DTTS data. The DTTS data consisted of 13000 images gathered over the course of the night at two second intervals; we estimated the LWE phase for each image and focus in particular on two subsets of this data: a strong LWE sequence over the course of one minute, and a strong LWE sequence over the course of one hour. The following statements below summarize our findings:

- Similar to our results in [2], single image Phase Diversity appears to be working using only the $20 \mathrm{~nm} \mathrm{rms}$ focus as our diversity.

- The estimation algorithm at minimum is a tool available to monitor the LWE; at the very least for P-V observations but it seems a single image is enough to fully characterize the system. Of course, if this technique was used in situ at the telescope, it would be desirable to put some known focus on the image and use traditional phase diversity in order to confirm this.

- We present the evolution of the LWE over a one-minute sequence. The particular sequence chosen shows some of the strongest LWE aberrations of the night, and even though we see a minor variation over the course of short timescales (i.e. one minute) this may not be reflective of all LWE cases. Considering the Peak-to-Valley WFE has a standard deviation of $62 \mathrm{~nm}$ over the one-minute squence, it seems if a correction were quantified every minute (with a corresponding update to the reference slopes with this estimate) then the LWE could be corrected to within this error.

- Evolution over a one-hour sequence: The 'episodic' nature of the LWE over the course of one hour further justifies the need for a quantification on the order of minutes as opposed to hours. The distinct nature of the three episodes shown in this work are not entirely known and may correspond to abrupt changes in wind direction (the wind speed is so slow that this is possible). However the determination of these features remains outside the scope of this work.

- Applying a correction: the algorithm requires only a few seconds to estimate the effect; as such an estimate and determination of offset slopes for the SHWFS is achievable many times per minute, and meets the requirements our suggestion of correcting at least once per minute be ideal.

- The applications Phase Diversity on high contrast imaging systems is an extremely desirable tool; as such we are exploring the capabilities of the technique developed here for the estimation of low order aberrations on the Gemini Planet Imager (GPI).

\section{ACKNOWLEDGMENTS}

The research leading to these results received the support of the A*MIDEX project (no. ANR-11-IDEX-0001-02) funded by the Investissements d'Avenir French Government program, managed by the French National Research Agency (ANR). This research was also funded in part by a MITACS/Campus France Globalink Research Award (ref: IT06712).

All the simulations and analysis done with the object-oriented MATLAB AO simulator (OOMAO) freely available from https://github.com/cmcorreia/LAM-Public

\section{References}

[1] Sauvage, J.-F., Fusco, T., Lamb, M., Girard, J., Brinkmann, M., Guesalaga, A., Wizinowich, P., O'Neal, J., N'Diaye, M., Vigan, A., Mouillet, D., Beuzit, J.-L., Kasper, M., Le Louarn, M., Milli, J., Dohlen, K., Neichel, B., Bourget, P., Haguenauer, P., and Mawet, D., "Tackling down the low wind effect on SPHERE instrument," in [Society of Photo-Optical Instrumentation Engineers (SPIE) Conference Series], Proc. SPIE 9909, 990916 (July 2016). 
[2] Masen P. Lamb, Carlos Correia, J.-F. S. J.-P. V. D. R. A. A. V. P. L. W. M. A. V. D. L. M. C. B., "Quantifying telescope phase discontinuities external to adaptive optics systems by use of phase diversity and focal plane sharpening," Journal of Astronomical Telescopes, Instruments, and Systems 3, 3-3-10 (2017).

[3] Vigan, A., Postnikova, M., Caillat, A., Sauvage, J.-F., Dohlen, K., El Hadi, K., Fusco, T., Lamb, M., and N'Diaye, M., "Characterisation of a turbulent module for the MITHIC high-contrast imaging testbed," in [Society of Photo-Optical Instrumentation Engineers (SPIE) Conference Series], Proc. SPIE 9909, 99093F (July 2016).

[4] N'Diaye, M., Dohlen, K., Fusco, T., and Paul, B., "Calibration of quasi-static aberrations in exoplanet direct-imaging instruments with a Zernike phase-mask sensor," A 6 A 555, A94 (July 2013).

[5] Gonsalves, R. A., "Phase retrieval and diversity in adaptive optics," Optical Engineering 21(5), 215829$215829-(1982)$.

[6] Paxman, R. G., Schulz, T. J., and Fienup, J. R., "Joint estimation of object and aberrations by using phase diversity," Journal of the Optical Society of America A 9, 1027-1085 (1992).

[7] Lamb, M., Correia, C., Sauvage, J.-F., Andersen, D., and Véran, J.-P., "Exploring the operational effects of phase diversity for the calibration of non-common path errors on NFIRAOS," in [Society of Photo-Optical Instrumentation Engineers (SPIE) Conference Series], Proc. SPIE 9909, 99096E (July 2016).

[8] Jolissaint, L., Mugnier, L. M., Neyman, C., Christou, J., and Wizinowich, P., "Retrieving the telescope and instrument static wavefront aberration with a phase diversity procedure using on-sky adaptive optics corrected images," in [Adaptive Optics Systems III], Proc. SPIE 8447, 844716 (July 2012). 